\title{
Filosofía, ciencia social y cognición humana: de la folk psychology a la psicología evolucionaria
}

\section{Jordi Mundó}

Universitat de Barcelona.

Departament de Teoria Sociològica, Filosofia del Dret

i Metodologia de les Ciències Socials

jordimundo@ub.edu

\section{Resumen}

Tradicionalmente, las ciencias sociales se han fundado en categorías de sentido común para describir la realidad, de modo que aceptan tácitamente el principio de la folk psychology, según el cual las personas actúan para conseguir aquello que desean, dadas unas creencias. Sin embargo, aunque los deseos y las creencias puedan ser causas de la acción, no hay modo de definir de forma conceptualmente independiente cada uno de estos elementos con el fin de elaborar leyes de la acción que sean informativas y empíricamente corregibles o ajustables. Se hace necesario substituir este sistema explicativo por otro que "divida la naturaleza por sus articulaciones». Alejada del fallido programa de investigación conductista, la ciencia social puede explorar nuevas vías para convertirse en una disciplina rigurosa equipada con un conjunto de teorías que permitan reorganizar las valiosas observaciones disponibles y sugerir nuevas hipótesis interdisciplinariamente integradas. Sin embargo, esto no ocurrirá en la medida en que no haya forma de escapar a las limitaciones de la folk psycho$\log y$. El presente artículo trata de mostrar cómo la psicología evolucionaria, centrada en los mecanismos evolucionados de procesamiento de información presentes en la mente humana, proporcionaría la conexión causal necesaria entre la biología evolucionaria y los complejos e irreductibles fenómenos sociales y culturales estudiados por sociólogos, economistas, antropólogos e historiadores.

Palabras clave: psicología evolucionaria, folk psychology, conductismo metodológico, ciencia cognitiva, filosofía de la ciencia social, causalidad.

\section{Abstract. Philosophy, social science and human cognition: from folk psychology to evolutionary psychology}

In the social sciences there has been almost universal agreement that the descriptive categories that common sense has used since the dawn of history are the right ones. Folk psychology tells us that people do the things they do roughly because they want certain ends and believe these acts will help attain them. However, beliefs and desires may be causes, but we can never find descriptions of them independent enough from one another to enable us to frame laws about them that have much informative content or improvable predictive power. We should replace this explanatory system with one that "carves nature at the joints». Far from the misguided and failed behaviorist research program, social science has an opportunity to turn itself into a theoretically rigorous discipline in which a powerful set of theories organize observations and suggest focused new hypotheses. This cannot hap- 
pen, however, as long as folk psychology continue to set the research agenda. The goal of this article is to clarify how evolutionary psychology, by focusing on the evolved information-processing mechanisms that comprise the human mind, supplies the necessary causal connection between evolutionary biology and the complex, irreducible social and cultural phenomena studied by sociologists, economists, anthropologists, and historians.

Key words: evolutionary psychology, folk psychology, methodological behaviorism, cognitive science, philosophy of social science, causality.

\section{Sumario}

1. Introducción: ciencia social con y sin psicología

2. La fuerza de la folk psychology y sus limitaciones

3. El conductismo metodológico y sus contradicciones
4. La psicología cognitiva y algunos de sus falsos supuestos

5. Psicología evolucionaria: una promesa de conexión causal

Referencias bibliográficas

\section{Introducción: ciencia social con y sin psicología ${ }^{1}$}

En las ciencias sociales hay un gran número de problemas sin resolver. En la tarea científica, de repente, por mor de un descubrimiento determinado, podemos empezar a hacernos nuevas preguntas que requieren de nuevas estrategias investigadoras. La física de altas energías, por ejemplo, pudo empezar a hacerse ciertas preguntas sobre la materia cuando se construyeron los aceleradores de partículas. Hoy, muchos biólogos creen que pueden responder a ciertas preguntas sobre la naturaleza humana, los orígenes de la especie y la naturaleza de la vida que habían sumido en la perplejidad a la ciencia y a la filosofía desde sus inicios.

El presente texto parte de la tesis de que algo así ocurre hoy en las ciencias sociales con las aportaciones de la psicología evolucionaria, la cual ha abierto caminos nuevos, ha corroborado hipótesis antiguas, ha puesto en la picota otras tradiciones académicamente muy arraigadas y ha contribuido a poner orden en ciertas discusiones que estaban en un callejón sin salida metodológico y conceptual. Muchas preguntas siguen sin respuesta, pero podemos aducir nuevas razones para sostener o refutar explicaciones que hasta ahora per-

1. Muchos de los aspectos desarrollados en el presente texto han sido discutidos en los cursos de doctorado que he impartido en los últimos años en la Facultad de Filosofía de la Universidad Nacional Autónoma de México, la Universidad Nacional de La Plata (Argentina), la Universitat Autònoma de Barcelona y la Universitat de Barcelona. Debo agradecer a los alumnos sus innumerables objeciones y sugerencias, que me han ayudado a perfilar y ordenar los problemas aquí presentados. Agradezco también a José Antonio Noguera que me haya animado pacientemente a ponerlo por escrito. El trabajo se inscribe en el proyecto de investigación HUM2005-03992, financiado por el Ministerio de Educación y Cultura, y el FEDER. 
manecían en el limbo de la ciencia. Ocurre, sin embargo, que, en ocasiones, hay una cierta renuencia a aceptar que las respuestas a ciertas preguntas de una disciplina puedan venir de otros campos.

Las preguntas tradicionales de las ciencias sociales reflejan la importancia de las elecciones de las hipótesis sobre las que discurrirá la investigación y sobre los métodos con que desarrollarla. Primero está la pregunta sobre si la acción humana puede explicarse del mismo modo en el que las ciencias naturales explican los fenómenos en su dominio. Las distintas respuestas posibles a esta pregunta llevan a subsiguientes preguntas: si es que sí, ¿por qué nuestras explicaciones sobre la acción humana son menos precisas y menos informativas que las explicaciones científicas de las ciencias naturales? Si la respuesta es que no, es decir, que los métodos de la ciencia natural son inapropiados, entonces ¿cuál es la forma correcta de explicar científicamente la acción? Y si no hay manera de explicar científicamente la acción humana - como defienden algunos filósofos y científicos sociales - ¿por qué la acción humana requiere un enfoque distinto del de la ciencia natural, y qué clase de enfoque requiere?

Estas preguntas son el equipaje de trasfondo de la ciencia social desde su origen y denotan que, generalmente, el tipo de pregunta que se hace el investigador social puede subdeterminar en gran medida el tipo de respuesta que obtendrá.

Supongamos, como estamos dispuestos a sostener aquí, que la ciencia social —incluso la que se ocupa de estudiar solamente variables agregadas — basa sus explicaciones económicas, sociológicas o antropológicas en ciertos supuestos sobre la psicología de los individuos. Esto quiere decir que, sea cual sea el campo social de análisis, por el mero hecho de estudiar la acción humana y sus consecuencias, siempre subyacen supuestos sobre la racionalidad, la propensión a la socialidad, la motivación o, en general, las dotaciones cognitivas de los humanos. En ocasiones es la propia teoría la que establece las precondiciones psicológicas sobre las que elabora las explicaciones sociales. Cuando esto ocurre, nos hallamos ante un caso relativamente sencillo de abordar, puesto que podemos examinar, poner en contradicción y, si procede, tratar de enmendar, la verosimilitud y la informatividad de los supuestos cognitivos inventariados. En cambio, resulta particularmente interesante para los fines del presente texto examinar en qué situación se halla la teorización social cuando los supuestos psicológicos sobre los que ésta se construye son implícitos y, a menudo, tácitos.

En este caso, pudieran darse dos circunstancias de especial interés: o bien suponemos la existencia de un acuerdo universal en la comunidad científica sobre la disponibilidad de una teoría psicológica suficientemente buena, lo cual permitiría tomarla como supuesto tácito de partida; o bien consideramos que la ciencia social es un ámbito gnoseológico autocontenido que no requiere hacer explícitos los principios procedentes de otras disciplinas, por ejemplo de la psicología. La primera opción presupone, a su vez, un reconocimiento de la necesidad de tener en cuenta las aportaciones de otras disciplinas, pero la común aceptación de una teoría haría redundante su incorporación explícita. 
La segunda interpretación niega de plano la bondad de un continuum epistémico y afirma a las ciencias sociales como reino autárquico.

En este texto me ocuparé básicamente de la primera interpretación. ¿Es realista pensar que el que en ciencia social no se haga explícita una descripción de la psicología humana tenga su razón en la aceptación general de una teoría psicológica suficientemente robusta e informativa? Puesto que en la psicología científica hoy por hoy no hay acuerdo en una teoría unificada, la respuesta debiera ser negativa. Pero cabe otra interpretación. En las ciencias sociales, una razón de peso para eludir predefiniciones psicológicas ha sido la aceptación de una tradición heredada, según la cual está fuera de discusión que las personas actúan para conseguir aquello que desean, dadas unas creencias. Se trata de una definición precisa y simple que presupone que el objeto de estudio de la ciencia social es la acción humana (ya sea individual o en agregados institucionales), y presupone asimismo que la acción humana está determinada por una conjunción de deseos y creencias que, a su vez, son razones y causas de la acción. Dicho en términos filosóficos, la persistencia de este esquema psicológico de fondo se explicaría porque cada uno de sus elementos da cuenta de una «clase natural» de hechos del mundo (la conducta humana y sus causas) y vendría avalado por su innegable eficacia predictiva en la vida social de los humanos.

\section{La fuerza de la folk psychology y sus limitaciones}

Los humanos navegamos con gran facilidad en el proceloso mar del mundo social por mor de la eficacia del simple esquema que conecta la acción humana con los deseos y las creencias. Desde la noche de los tiempos, los humanos somos capaces de entender de un modo sorprendentemente sencillo la acción de alguien si conocemos cuáles eran sus creencias y cuáles los deseos que le animaron. En otro sentido, podemos predecir con gran fiabilidad cuál va a ser la acción que emprenderá otra persona si de antemano conocemos cuáles son sus deseos y creencias. Más aún, conociendo cuál ha sido su acción y cuáles sus deseos, podemos inferir verosímilmente qué creencias albergaba. Y, en fin, sabiendo cuál ha sido su acción y cuáles sus creencias, podemos conjeturar muy aproximadamente cuáles eran sus deseos. En lenguaje de filosofía de la mente, este esquema es conocido como folk psychology o psicología intuitiva o de sentido común. Se trata de una supuesta explicación de la naturaleza psicológica humana que funciona con extraordinaria precisión en las interacciones sociales entre los miembros de la especie ${ }^{2}$.

De aceptar que las categorías descriptivas de sentido común de la folk psychology son correctas, es decir, de aceptar que las acciones, los deseos y las creencias son descripciones que, como diría Aristóteles, dividen las diferentes partes de la realidad por sus articulaciones, la pregunta relevante sería si la folk

2. Para una presentación de los principios de la folk psychology, véanse, por ejemplo, Dennett (1987; 1991), Goldman (1993), Stich y Nichols (1992) y Putnam (1979). 
psychology puede coadyuvar a una explicación causal de los mecanismos subyacentes de la acción humana. Los humanos depositamos una gran confianza en la folk psychology porque nos permite hacer inferencias, predictivas o retrodictivas, que resultan empíricamente muy fiables. Se trata de un esquema que utilizamos de una forma inconsciente, al igual que ocurre con la gramática que regimenta nuestro habla, la cual evita que cometamos errores sintácticos básicos sin que necesariamente tengamos consciencia de ello. Si de esto dedujéramos que las explicaciones folk psychology funcionan muy bien como explicaciones causales de la acción humana, que acotan y dan cuenta plausiblemente de la intencionalidad humana, podríamos llegar a pensar sin violencia conceptual que la folk psychology es una excelente candidata para dar forma a las explicaciones en ciencias sociales. Entonces, nos preguntaríamos, ¿por qué no dejar que la ciencia social trate de desarrollarse a partir de explicaciones folk, si resulta que éstas nos garantizan un éxito predictivo tan estimable? Se trataría de ampliar esta "teoría» a partir de la comprensión de las interacciones de los individuos hasta llegar a la modelización de las interacciones que ocurren en las instituciones sociales entre un gran número de individuos, y también hasta las interacciones entre individuos cuyas culturas y formas de vida son distintas de las propias.

Más que una mera hipótesis, es un hecho que una parte importante de la ciencia social se ha desarrollado a partir de un esquema folk, a menudo sin tener plena consciencia de ello ${ }^{3}$. Regiones relevantes de la ciencia social han tomado la folk psychology como un mecanismo de descubrimiento de significados, de comprensión de los hechos sociales humanos, de vía por la que se puede hacer inteligible la acción humana, de modo que han permitido desechar explicaciones causales fuertes — en el sentido de las que se pretenden en las ciencias naturales. En este sentido, que conecta con la venerable tradición de la hermenéutica y las Geisteswissenchaften (o ciencias del espíritu, en oposición a las Naturwissenchaften, o ciencias naturales), el propósito de las ciencias sociales no sería el de explicar —en sentido causal fuerte-, sino el de comprender — de algún modo explicar, utilizando las razones como causas de la acción-, el de hacer inteligible la acción. Cabe preguntarse, por tanto, si la economía, la sociología, la antropología, la ciencia política o la historia tienen a su disposición — vía folk psychology - una teoría causal de la acción humana en sentido fuerte, o si sólo pueden aspirar a utilizarla para hacer inteligible la acción humana.

Lo normal en ciencia empírica a la hora de establecer una conexión causal (fuerte) entre $A$ y $B$ es disponer de descripciones de $A$ y de $B$ que sean conceptualmente independientes del vínculo causal existente entre ellas. Cuando afirmamos, por ejemplo, que hay un vínculo funcional y causal entre la presión de un gas, su volumen y su temperatura, tenemos medios de controlar todas esas variables (de describirlas y de medirlas) de un modo conceptual- 
mente independiente de la ley de Boyle, que expresa el vínculo funcional (y causal) entre ellas. Eso es lo que hace que esa ley sea, en principio, corregible y ajustable empíricamente. En cambio, no parece fácil encontrar descripciones de las creencias, los deseos y las acciones de los individuos que sean conceptualmente independientes entre sí y conceptualmente independientes del esquema básico o «ley» de la folk psychology. Parece difícil imputar deseos y creencias que expliquen la acción de alguien de un modo conceptualmente independiente de la hipótesis (esa hipótesis es la «ley» de la folk psychology) de que ese alguien es coherente o racional en algún sentido que nos haga inteligible su acción. El problema radica en que la peculiar noción de causalidad comprensiva (utilizar las razones de la acción como causas de la acción) tiene su talón de Aquiles en que la «ley» de la folk psychology no sería interpretable causalmente en ningún sentido filosófico serio; esa, digamos, «ley», sería empíricamente incorregible, y sólo serviría para hacernos inteligible la acción humana, para hacernos comprenderla, no explicarla. Y la imposibilidad de corrección empírica haría que las hipótesis resultantes corrieran el serio riesgo de ser vacuas o arbitrarias. El circulo hermenéutico formado por deseos, creencias y acciones sólo permitiría, pues, explicaciones fatalmente circulares ${ }^{4}$.

De modo que toda la fuerza de la folk psychology, su innegable poder para realizar inferencias sobre los estados mentales intencionales de los individuos en las interacciones cotidianas, se vuelve contra ella cuando se pretende extenderla a otros ámbitos de la teorización social que sobrepasen el nivel folk, intuitivo o de sentido común.

De todo esto podría desprenderse que, o bien optamos por considerar que la ciencia social es un ámbito gnoseológico autocontenido que no requiere hacer explícitos los principios ni la metodología de investigación procedentes de otras disciplinas (una suerte de reino causalmente aislado), de modo que no nos quedaría otra salida que la vía de exploración hermenéutica, en alguna de sus innumerables ramificaciones; o bien, si seguimos considerando epistémicamente irrenunciable la necesidad de encontrar explicaciones causales empíricamente corregibles y consideramos, además, que para conseguirlo es condición necesaria que la ciencia social tenga en cuenta los resultados de las ciencias adyacentes, entonces debemos optar por reconceptualizar la relación entre la ciencia social y la psicología 5 .

\section{El conductismo metodológico y sus contradicciones}

Uno de los intentos más logrados en términos académicos de esta segunda línea, digamos, analítica vino de la mano del llamado «conductismo». El conductismo nace en gran medida como una respuesta a los problemas filosóficos y metodológicos que plantea la explicación intencional. Al tomarse muy

4. Para un interesante argumento funcionalista de la incorregibilidad de la «teoría» folk, véase Putnam (1964).

5. Sobre el separatismo en ciencia, cfr. Mundó (2001; 2002). 
en serio el problema de las «otras mentes» (la cuestión sobre cómo podemos conocer los estados mentales aparentemente privados de los demás sin disponer de otro dato que su conducta), se propone abordar los problemas de la actividad social humana sorteando las implicaciones intencionales $\mathrm{y}$, en general, cognitivas de la misma.

Para los conductistas, las explicaciones de la folk psychology tienen una apariencia de causalidad que esconde lo que no es más que una explicación teleológica. No habría modo de identificar creencias y deseos en términos de estados cerebrales independientemente de sus efectos, las acciones. Deseos y creencias tendrían la apariencia de distintas causas previas a la acción, pero, puesto que no podrían disociarse suficientemente de sus efectos futuros, no se podría dar cuenta de los mismos de un modo realmente informativo. Entonces, arguyen los conductistas, si este tipo de explicaciones no son informativas, no pueden ser causales, puesto que el sello identificador de una explicación causal es su capacidad para aportar información fáctica sobre sucesos no triviales ${ }^{6}$.

El conductismo psicológico o metodológico — distinto del conductismo filosófico u ontológico- ${ }^{7}$ no niega la existencia de una mente (e incluso puede llegar a reconocerle una notable complejidad), sino que opta por el estudio no mental de la conducta como fenómeno unificador de las ciencias sociales. Las preguntas sobre la mente serían despreciables por al menos uno de estos tres argumentos metodológicos: primero, porque la conducta humana puede explicarse sin necesidad de referirse a la mente; segundo, porque la conducta humana en realidad no puede explicarse recurriendo a categorías mentales, y, tercero, porque las preguntas sobre la mente humana son en sí mismas imposibles de responder. El conductismo psicológico no sólo critica la incapacidad de la folk psychology para realizar explicaciones causales fuertes, sino que pretende combatir por entero las explicaciones intencionales, las cuales, sostiene, a pesar de su probado extravío, siempre han constituido, al menos desde Aristóteles, la última barricada de la estrategia de investigación en ciencia social ${ }^{8}$.

El conductismo, sobre todo a partir de Skinner, supone que el objeto de la ciencia no es la comprensión de la mente o la descripción compleja de la

6. Sobre los problemas de las explicaciones teleológicas en el conductismo, véanse los textos clásicos de Cummins (1975), Hempel (1965), Nagel (1961, capítulo 12), Rudner (1966), Taylor (1964) y Wright (1976).

7. El conductismo ontológico sostiene que, puesto que toda psicología es conducta, y nada más que conducta, cualquier afirmación de algún hecho supuestamente mental no es más que una explicación de la conducta basada en conceptos erróneos. Para el conductismo ontológico, no existe una mente distinta de la conducta en ningún sentido relevante (cfr. Sober, 1985).

8. Asunto aparte, aunque nada baladí, es el que tiene que ver con que la opción metodológica de explicar la vida social humana sin recurrir a categorías mentales, aunque formalmente no conlleve la negación de la existencia de la mente humana, en realidad significa situar la mente en una condición de irrelevancia extrema que deriva, lógicamente, en un conductismo ontológico: de tanto negar — metodológicamente- relevancia a la mente, se acabaría infiriendo, por el desideratum de parsimonia de la argumentación, la negación de la existencia de estados mentales de cualquier tipo (cfr. Sober, 1985, p.182). 
psicología humana, sino la sistematización del comportamiento observable. Sistematizar el comportamiento significa proporcionar explicaciones generales que nos permitan correlacionar las condiciones ambientales observables con el comportamiento que éstas inducen ${ }^{9}$. De lograrlo, habría una vía abierta para bosquejar explicaciones causales en sentido fuerte, puesto que se estaría en condiciones de definir de forma independiente cada uno de los elementos del sistema, y éstos de la ley general, lo cual, a su vez, permitiría corregir y ajustar la teoría. Miel sobre hojuelas, reduciendo la actividad humana a «mero» comportamiento, se habría salvado el escollo de la circularidad en la que se enzarza la explicación folk.

La estrategia conductista ha tenido un reconocido predicamento en ciencias sociales como la sociología, la antropología o la ciencia política, no tanto porque haya habido ramas de estas ciencias que se hayan adscrito abiertamente a ese programa de investigación, sino porque implícitamente han incorporado el mayor de sus supuestos, a saber, que puede hacerse buena teoría social sin necesidad de apelar a descripciones de la psicología humana. Acaso de forma más sorprendente, esto también ha ocurrido en la teoría económica llamada «estándar» o "neoclásica». Sorprendente porque se supone una verdad de Perogrullo que la teoría de la elección racional en la que se basa la teoría económica neoclásica no es otra cosa que una formalización sofisticada de la folk psychology. La asunción de este supuesto, entre otros menores, ha dado pie a la tradicional acusación realizada a la teoría económica estándar de ser o bien falsa, o bien vacua y no sometible a prueba. De entre las distintas estrategias para evitar estos cargos, una de ellas consistiría en reinterpretar el objeto de la teoría económica excusándola de tener que explicar la acción humana individual, y asignándole la tarea de explicar el «mero» comportamiento. Esta reinterpretación está en la base, por ejemplo, de la teoría de la "preferencia revelada". Aunque la teoría económica estándar históricamente había sustituido ya la utilidad cardinal por su versión matemáticamente más manejable de la utilidad ordinal, a decir de muchos economistas aún se trataba de una teoría demasiado psicológica ${ }^{10}$. El desideratum sería evitar hacer suposiciones sobre qué ocurre en el interior de las cabezas de los agentes. Con la utilización del lenguaje

9. Para la más difundida defensa del conductismo, véanse Skinner (1953; 1974). Se puede encontrar una provechosa introducción a la psicología experimental de influencia skinneriana en Rachlin (1970).

10. Por razones que escapan al propósito del presente texto, la teoría económica neoclásica abandonó la cardinalidad de las preferencias. La utilidad cardinal no sólo refleja la ordenación de preferencias de la utilidad ordinal, sino que además mide la intensidad de esas preferencias. Puede encontrarse una excelente historia de los cambios en la teoría de la utilidad en Blaug (1997). Para entender algunas de las razones por las que, por ejemplo, la escuela austríaca desechó por demasiado «subjetivo» el enfoque de un Carl Menger (1981) preocupado por conectar los deseos humanos con variables biológicas y fisiológicas, véanse Hayek (1973) y Von Mises (1981); para una ilustración del conflicto de la escuela austríaca tardía con los supuestos «demasiado psicológicos» de la teoría económica — no estándar- de Keynes, véase Butos (2003). 
de las preferencias reveladas (no internas, no inferidas de estados intencionales, sino manifiestas), supuestamente se conseguiría que el único criterio normativo que debiera respetarse fuera el de la consistencia de la conducta, no sus causas psicológicas. Esta formulación no es más que una variante de conductismo metodológico. Pero la realidad es proteica y la utilización de estrategias científicas procrusteanas para evitar tener que lidiar con la complejidad de los fenómenos a estudiar suele llevar a vías muertas. Los endebles supuestos de partida de la teoría de las preferencias reveladas restringieron aún más el campo propio de la teoría económica - ya muy menguado con el paso de la cardinalidad a la ordinalidad de la utilidad-, de modo que se exploraron caminos menos triviales, como la teoría de la «utilidad esperada» de Von Neumann y Morgenstern (1953), que permitió un regreso por vía rodeada al potencial de la utilidad cardinal - aunque sin posibilidad de comparaciones interpersonales-y una reincorporación de las variables de deseo, creencia y acción. El que la teoría económica rescatara variables intencionales no es nada casual. A pesar del estimable empeño del conductismo metodológico de arrasar con el lenguaje intencional de la folk psychology, resulta a la vez paradójico y revelador descubrir que uno de los mayores fracasos del conductismo - además del de no rebasar la eficacia predictiva de la folk psychology - tiene que ver precisamente con su incapacidad para eliminar por completo el lenguaje y los supuestos intencionales ${ }^{11}$. Paradójico porque el camino seguido para alcanzar su propósito se torna inconsistente; y revelador porque permite conjeturar verosímilmente que la explicación de la acción humana tiene, por la naturaleza de ésta, componentes irreductiblemente intencionales que se resisten a teorizaciones que nieguen su existencia. Lo interesante es que - tenga las limitaciones que tenga la teoría de la «utilidad esperada» y, en general, la teoría económica neoclásica- queda patente que cualquier intento de eludir una descripción de las psicologías humanas compleja y realista parece abocado a pseudoexplicaciones causales ${ }^{12}$.

Podría resumirse lo visto hasta aquí diciendo que, por un lado, las ciencias sociales heredan una inveterada tradición psicológica, según la cual los humanos estamos dotados de una psicología intuitiva, o folk psychology, que nos permite navegar con facilidad por la vida social, de modo que podemos atribuir estados mentales intencionales a los demás y hacer predicciones e inferencias muy fiables sobre su comportamiento. La utilización de esta disposición como "teoría» extensible a las explicaciones de toda la vida social individual y colectiva, empero, no permitiría realizar explicaciones causales en sentido fuerte, porque no hay modo de definir de forma conceptualmente independiente cada uno de

11. Cfr. Horgan y Woodward (1985) y Rosenberg (1988, p. 62-65).

12. Sobre las limitaciones de la teoría económica neoclásica en relación con la psicología que presupone, véanse: Domènech (2002), Hausmann (1992), Rabin (1994), Rosenberg (1992), Simon (1986) y Thaler (1987). Para un análisis sofisticado de las implicaciones para la ciencia social que puede tener una concepción de la psicología humana como mera colección de disposiciones intencionales atomizadas, véanse Pettit (1993) y Domènech (2005). 
los elementos de la explicación, ni éstos independientemente de la "ley» general, de modo que se incurre en el riesgo de explicaciones falsas, vacuas o arbitrarias. Y hemos visto, por otro lado, que el conductismo metodológico trataría de sortear el riesgo de vacuidad o arbitrariedad de las explicaciones intencionales utilizando un esquema explicativo que supuestamente favorecería las explicaciones causales en sentido fuerte a partir de explicaciones generales que permitirían correlacionar las condiciones ambientales observables con el comportamiento que éstas inducen. Pero la dramática restricción que impone a los supuestos de partida la eliminación de cualquier variable psicológica limita enormemente la relevancia de las explicaciones alcanzables de la acción humana. Además, parece que la propia estrategia conductista, no independientemente de la razón anterior, difícilmente puede extirpar como pretendía todo lenguaje intencional.

\section{La psicología cognitiva y algunos de sus falsos supuestos}

El conductismo skinneriano, como va dicho, ha sido una de las manifestaciones institucionalmente más exitosas de cierta forma de entender la psicología, pero su antimentalismo y su cientificismo doctrinario lo hizo implausible para aquéllos que buscaban una explicación de la experiencia interna ${ }^{13}$. Además, su énfasis en las historias individuales de refuerzo limitó las explicaciones del complejo fenómeno de la cultura humana, sus mecanismos de creación, transmisión y selección. Todo esto constituyó un blanco fácil cuando la ciencia cognitiva proporcionó formas más precisas de caracterizar e investigar lo mental entendiéndolo como un sistema de procesamiento de información, una caracterización que abría nuevas líneas de aproximación a las motivaciones de la acción humana más interesantes que las tablas de refuerzo conductistas. Simbólicamente, el comentario crítico de Chomsky (1959) al famoso texto de Skinner Verbal Behavior (1957) marcó un punto de inflexión en el programa de investigación conductista. Recuérdese que uno de los puntos de apoyo teóricos del conductismo psicológico consistía en su explicación pretendidamente en sentido causal fuerte- del lenguaje humano por un proceso propio del esquema básico del conductismo operante ${ }^{14}$. La supuesta explicación causal de cómo un bebé que nace sin capacidad lingüística acaba siendo un hablante competente de una lengua particular a partir de la recepción y el refuerzo de estímulos lingüísticos fue puesta en entredicho por Chomsky, con el argumento de que esta teoría no permitía explicar por qué los niños de temprana edad son capaces de elaborar ciertas construcciones verbales que nunca han oído anteriormente. La única explicación verosímil, sostenía Chomsky, pasaría por suponer que los humanos nacen dotados de

13. Tienen particular interés las críticas al cientificismo doctrinario de Skinner realizadas por Chomsky (1975), Dennett (1981) y Flanagan (1991).

14. El conductismo operante sigue el siguiente esquema: «si un comportamiento es reforzado, se repetirá con mayor frecuencia (o intensidad o duración). Si es penalizado, se repetirá con una frecuencia (o intensidad o duración) menor». 
una capacidad innata para el lenguaje, que desarrollamos en un contexto de interacción social simbólica. De ser cierta esta observación, para explicar la actividad humana sería insuficiente contar sólo con las variables ambientales observables y habría que tener en cuenta, necesariamente, elementos internos de la psicología humana, en particular su estructura cognitiva ligada a los contenidos lingüísticos.

De modo que el argumento de la existencia de mecanismos cognitivos específicos para el dominio lingüístico llevaba implícito el ofrecimiento de un cambio radical en la concepción de la mente humana. Y la nueva ciencia cognitiva parecía ser el vehículo de esa transformación. Sin embargo, repárese en que el elemento clave de falsedad de la argumentación conductista era el de que, al suponer que podían realizarse explicaciones fiables de la actividad humana eludiendo cualquier referencia cognitiva, estaba a su vez suponiendo que la mente desempeña un papel neutral en la mediación entre los estímulos recibidos y la conducta exhibida. De lo cual se infiere que el conductismo metodológico concibe por defecto la mente como una tabula rasa, un recipiente inicialmente vacío carente de mecanismos específicos para discriminar los distintos tipos de estímulos que recibe del exterior ${ }^{15}$. Dicho en el lenguaje de la ciencia cognitiva, el conductismo presupone que la mente tiene una configuración estructural independiente de cualquier patrón de información que potencialmente pueda procesar. Sólo existirían mecanismos de uso general, equipotenciales, de modo que cualquier descripción psicológica sería dependiente de los conceptos «externos» de aprendizaje y socialización. En fin, los humanos desarrollaríamos estructuras cognitivas - se entendiera lo que se entendiera por tales - a partir del supuesto implícito de existencia de socialización masiva. La aparición de la psicología cognitiva puso en evidencia, precisamente, que esto era falso: existirían — como en el caso del lenguaje - algunos mecanismos innatos que interaccionarían con las variables sociales o culturales, de modo que nociones tales como socialización masiva o construcción social de la realidad, tan comunes en la ciencia social, deberían ser seriamente reconsideradas ${ }^{16}$.

Pues bien, con la emergencia de la ciencia cognitiva, parecía probado que la mente no podía ser una tabula rasa, sino que contenía algunos mecanismos innatos dominio-específicos. Pero eso, interesantemente, no condujo a abrir una línea principal de investigación que tuviera como hipótesis de partida, por ejemplo, que la mente es un procesador de información equipado por un amplio espectro de mecanismos especializados. De modo que en muchos aspec-

15. Para una introducción muy pedagógica sobre la noción de tabula rasa cognitiva, véase Pinker (2002).

16. El texto quizá más representativo de esta concepción sea el de Berger y Luckmann (1967). Es preciso no olvidar que, significativamente, gran parte de la antropología en la que se apoyan las tesis de los autores está inspirada, entre otras, en concepciones tan extremas sobre la maleabilidad de la naturaleza humana como las de la antropología filosófica de su maestro Arnold Gehlen (1966). 
tos el cambio metodológico y conceptual que trajo consigo la ciencia cognitiva no cuestionó por completo los resultados del programa conductista ${ }^{17}$. La razón de la convergencia entre ambos programas estriba en que, a pesar del rechazo de los psicólogos cognitivos del incómodo antimentalismo conductista, en general adoptaron acríticamente el supuesto de la equipotencialidad, limitando así dramáticamente el horizonte analítico de las nuevas líneas de investigación. En el mainstream de la psicología cognitiva o científica, se suponía que la mente está formada básicamente por mecanismos de uso general, no por mecanismos especializados en el procesamiento de información diferenciada (con la excepción de algunos dominios muy acotados). Los científicos cognitivos justificaban este supuesto apelando al principio de parsimonia: es «acientífico» multiplicar los hipotéticos mecanismos en la mente humana; el objetivo, como en física, es describir con los mínimos principios la mayor complejidad posible.

Así que, con la refutación del conductismo, ocurrió un cambio metodológico que conllevó un cambio de formulación: los humanos pasarían de ser vistos como sistemas (conductistas) de aprendizaje (operante) equipotencial relativamente simples a sistemas complejos de procesamiento de información equipotencial, ordenadores de procesamiento general o sistemas manipuladores de símbolos, que además estaban dotados de algunos dominios especializados $^{18}$. Con la excepción de ciertos módulos específicos que podían definirse como cápsulas relativamente autónomas, la mente siguió concibiéndose como un mecanismo de uso general casi ilimitadamente moldeable por el entorno $^{19}$, de modo que los conceptos de aprendizaje, socialización, mecanismos de uso general (o independientes del contenido) han seguido dominando la psicología científica durante los últimos sesenta años ${ }^{20}$.

Esta concepción paraconductista de la psicología humana ha proporcionado los supuestos psicológicos básicos — tácitos la mayoría de las veces- de muchas líneas de investigación dominantes en ciencias sociales ${ }^{21}$. Reinterpretado

17. Además, en algunos aspectos, perduraron ciertas inercias programáticas: «Sorprendentemente, los científicos cognitivos empezaron compartiendo con los conductistas el supuesto de que existía algo parecido a una ley de las correspondencias entre estímulos y respuestas» (Flanagan, 1991, p. 177).

18. Véase, por ejemplo, Sahlins (1976a, 1976b).

19. Lo cual ayuda a entender la persistencia de líneas de investigación como el conexionismo. Cfr. Karmiloff-Smith (1992).

20. Se calla por sabido que en psicología existen algunas comunidades de investigadores que no conciben así la mente humana y que han estado más consistentemente conectadas con el resto de la ciencia, como los campos que se ocupan de la psicología fisiológica, la percepción, la psicofísica, la motivación, la psicolingüística, gran parte de la psicología comparada y otras áreas. Para una introducción conceptual y epistemológica de los inicios de la psicología cognitiva, véanse Dennett (1995) y Rosch (1997).

21. Cualquier generalización es arriesgada, porque deja fuera decenas de sistemas intelectuales cuidadosamente desarrollados que contienen genuinas diferencias entre ellos. Pero el propósito del presente texto es el de mostrar algunas de las concepciones dominantes en ciencias sociales, lo cual obliga a omitir la discusión sobre un gran número de comunidades 
programáticamente, este enfoque mostraría la siguiente relación bidireccional entre la ciencia social y la cognición humana:

1) La constatación empírica de la diversidad de las conductas humanas supone una negación implícita de la importancia de hipotéticos mecanismos psicológicos evolucionados;

2) de lo cual se infiere que, puesto que la aparentemente "constante» naturaleza cognitiva infantil no puede explicar la enorme «variedad» de la psicología adulta, entonces, la "naturaleza humana» (o su supuesta concepción moderna, la estructura evolucionada de la mente humana) no puede ser causa de la organización mental de los humanos adultos, sus sistemas sociales, su cultura, sus procesos de cambio histórico, etc.

3) Puesto que la organización mental adulta (pautas de conducta, conocimiento, realidades socialmente construidas, etc.) no está presente en la psicología de los niños, se infiere que, durante su proceso de desarrollo, éstos la «adquirirán» a partir de alguna fuente externa.

4) La organización mental a incorporar está obviamente presente en el mundo social en forma de conducta y de representaciones públicas de los demás miembros del grupo local. De este modo, la composición de lo mental puede caracterizarse haciendo una clara distinción: por un lado, estaría lo «innato» (lo genéticamente determinado, lo biológico), que no es negado, y que constituye lo observable en la infancia; por otro lado, estaría lo social (o cultural, o aprendido, o adquirido, o ambiental), que comprendería todo lo organizado de forma compleja y que sería suministrado por el entorno social (con algunas excepciones, también proporcionado por el entorno físico y el aprendizaje no social).

5) De donde se deduciría que el mundo social sería la causa de la organización mental de los adultos. Así, se supone que todos los elementos «culturales» que moldean al individuo lo preceden, y son externos a él. La mente no los crea: ellos crean la mente.

6) De acuerdo con esto, lo «social» o «cultural» es el organizador que dota de complejidad y da forma a la sustancia de la vida humana. Este acervo («exosomático», "extragenético») es diversamente describible como conducta, tradiciones, conocimiento, símbolos significativos, hechos sociales, sistemas semióticos, información, organización social, relaciones sociales, relaciones económicas, mundos intencionales o realidades socialmente construidas.

7) Todo ello lleva a la negación de la posibilidad de que la cognición humana pueda desempeñar algún papel relevante en la organización de la vida social humana, y supone una delimitación del rol de la arquitectura de

disidentes dentro de la sociología, la antropología, la economía y otras tantas disciplinas, que no han adoptado una concepción de la mente humana equipotencial e independiente de su contenido. En el mundo real, no existen los tipos ideales. La crítica va dirigida a una forma de pensar, no a la miríada de trabajos de investigación concretos. 
la mente humana a mera capacidad vacía para la cultura y la sociali$\mathrm{dad}^{22}$.

8) Entonces, la psicología en la que se funda la ciencia social es la disciplina que estudia los procesos de socialización y el conjunto de mecanismos de esa capacidad $^{23}$. Lo cual implica que el concepto central en psicología debe ser el de «aprendizaje».

9) Todo componente, proceso o mecanismo cognitivo debe ser equipotencial, independiente del contenido, de uso general, de dominio general, etc.

De modo que, con todas las correcciones y matices que se quiera, el salto del conductismo metodológico a la psicología cognitiva fue muy deudor de una metaconcepción dualista propia de nuestra tradición cultural $-\mathrm{y}$, particularmente, científica-, que limitó la exploración de una descripción fiable de la psicología humana independiente de sus presupuestos socioantropológicos, y al revés. Esta concepción es la extensión del dualismo de las dicotomías material-espiritual, cuerpo-mente, físico-mental, natural-humano, biológicosocial, biológico-cultural, racionalismo-empirismo, herencia-entorno, instinto-aprendizaje, naturaleza-educación, universales humanos-relativismo cultural, naturaleza humana-cultura humana, conducta innata-conducta adquirida, inflexible-maleable, esencialismo-construcción social, etc.

Los científicos sociales que inicialmente prestaron atención a la neurociencia, la etología y la psicología cognitiva cada vez fueron más conscientes de la evidencia de que el sistema cognitivo no podía ser equipotencial en sentido conductista. No obstante, raramente fueron más allá del mero reconocimiento a beneficio de inventario de que la noción de tabula rasa era insostenible. Generalmente, el lenguaje de la tabula rasa fue substituido por la jerga del procesamiento de información consistente en procesos cognitivos vacíos ${ }^{24}$.

22. Quizá no haya en la literatura ejemplo mejor de lo que significa una concepción sociológica de una psicología vacía que sólo incorpora la "capacidad» para la socialidad que este famoso paso de Durkheim (1987/1895, p. 117-118): «Pero se engañaría quien de lo que antecede quisiera sacar la conclusión de que, en nuestra opinión, la sociología debe, y hasta puede, hacer abstracción del hombre y sus facultades. Es por el contrario evidente que los caracteres generales de la naturaleza humana entran en el trabajo de elaboración de donde resulta la vida social. Únicamente, que no son ellos quienes la suscitan, ni quienes le dan una forma especial: únicamente la hacen posible. Las representaciones, las emociones, las tendencias colectivas, no tienen por causas generatrices determinados estados de la conciencia de los particulares, sino las condiciones en que se encuentra el cuerpo social en su conjunto. Claro está que no pueden realizarse si las naturalezas individuales les son refractarias, pero éstas no son más que la materia indeterminada que el factor social determina y transforma. Su contribución consiste exclusivamente en estados muy generales, en predisposiciones vagas y, por consiguiente, plásticas, que por sí mismas no podrían tomar aquellas formas definidas y complejas que caracterizan los fenómenos sociales, si no intervinieran otros agentes».

23. Spuhler (1959).

24. Para una exposición iluminadora de las diversas «herramientas» (desde el trozo de cera hasta los ordenadores de procesamiento general, pasando por la inferencia estadística) que han sido utilizadas como metáforas para dar cuenta de la misma estructura de la mente humana, véase Gigerenzer (1991). 
¿Quiere esto decir que, una vez aprendidas las lecciones de la fallida aventura conductista, en la caracterización de la psicología y de sus consecuencias para el análisis social estamos abocados a movernos entre el fuego de la psicología folk y las brasas de cualquier alternativa basada en una psicología científica, no intuitiva?

\section{Psicología evolucionaria: una promesa de conexión causal}

La ciencia cognitiva no puede tener una agenda de investigación guiada por la folk psychology. Los resultados empíricos de las últimas décadas de la biología evolucionaria, la inteligencia artificial, la psicología del desarrollo y la lingüística, entre otras, ofrecen a la psicología cognitiva la oportunidad de superar las limitaciones de un enfoque autocontenido. La ciencia cognitiva dispone de la alternativa de fundar sus teorías en supuestos evolucionarios rigurosos que den cuenta de cómo la mente humana adquirió su organización funcional durante el proceso evolutivo. Las teorías biológicas funcionales adaptativas describen el tipo de problemas para los cuales nuestra mente fue diseñada por la evolución, y proporcionan una información esencial sobre qué rasgos de diseño es probable que tengan estos mecanismos. Esta clase de información procedente de otras ciencias adyacentes libera a los científicos cognitivos de las limitaciones de la pura intuición y la folk psychology, y les permite construir experimentos capaces de detectar mecanismos complejos que de otro modo ni siquiera se les habría ocurrido tratar de $\operatorname{probar}^{25}$.

Los resultados de las ciencias antes mencionadas han cristalizado en la psicología evolucionaria. La psicología evolucionaria es simplemente psicología informada por el conocimiento adicional de la biología evolucionaria, que parte de la hipótesis de que la comprensión del proceso que diseñó la mente humana permitirá saber cómo es su arquitectura y con ello coadyuvará a alumbrar una descripción más realista de la cognición y la psicología humanas. Esta descripción realista, centrándose en los mecanismos evolucionados de procesamiento de información que forman parte de la mente humana, esboza un sendero de conexión causal entre la biología evolucionaria y los complejos e irreductibles fenómenos sociales y culturales estudiados por sociólogos, antropólogos, economistas e historiadores ${ }^{26}$. Las realidades sociales y culturales no se explican sólo por causas sociales o culturales: no son ajenas a los mundos físico, biológico o cognitivo. La socialidad y la

25. Cfr. Cosmides y Tooby (1994a) y Atran (2005). Rosenberg (1988, p. 89) señala: «Nuestra folk psychology y la de los demás alcanza cierto horizonte de precisión predictiva, pero allí se detiene. Un enfoque naturalista, que busca conocimiento causal, no puede detenerse en la folk psychology, sino que debe continuar exigiendo mejoras en la capacidad de predicción. Tales mejoras constituyen las trazas de futuros descubrimientos sobre las causas de la conducta».

26. Para una introducción de la importancia de la psicología evolucionaria para la ciencia social, cfr. Barkow y otros (1992), Mundó y Raventós (2000) y Mundó (2003). 
cultura son generadas de formas muy ricas e intricadas por mecanismos de procesamiento de información que están situados en las mentes humanas. Estos mecanismos son, a su vez, el producto esculpido por miles de años del proceso evolutivo. Entonces, para entender la relación entre biología y socialidad, o entre biología y cultura, hay que comprender la arquitectura evolucionada de nuestra cognición. Intentos anteriores de aplicar la biología evolucionaria directamente a la vida social humana no siempre han sido atinados, precisamente porque no han prestado suficiente atención a este aspecto esencial ${ }^{27}$.

La promesa que lleva consigo la psicología evolucionaria es la conexión causal entre el mundo biológico y el psicológico, y entre éste y el social, un ambicioso programa de investigación que implica consistencia entre campos. Para ello es prescriptivo el abandono de las dicotomías simplificadoras entre lo social y lo natural, lo natural y lo humano, etc. que han constreñido por décadas a la ciencia cognitiva. Puede resumirse la crítica a la concepción tradicional en tres aspectos principales.

En primer lugar, el modelo de psicologías vacías se basa en un análisis desacertado de lo innato y lo aprendido. Existe una apreciación incorrecta del rol que desempeña la evolución en la regimentación de la relación existente entre la dotación genética humana universal, sus procesos de desarrollo evolucionados y los rasgos recurrentes de los entornos en los que se produce tal desarrollo. Para tomar uno de entre los muchos errores conceptuales presentes en la literatura psicológica, obsérvese que la idea de que el fenotipo puede dividirse dicotómicamente en rasgos genéticamente determinados y rasgos determinados por el entorno es palmariamente incorrecta. Un modelo general que suponga que los «factores biológicos» y los «factores ambientales» se refieren a conjuntos mutuamente excluyentes de causas existentes en cierto tipo de relación explicativa de suma cero (cuanto más se explica algo biológicamente, menos margen queda para explicarlo «social» o «ambientalmente») es erróneo: de hecho, una explicación «ambientalista» consistente requiere la existencia de una rica arquitectura evolucionada. Por lo mismo, la premisa básica de la oposición entre la mente entendida como un producto biológico inflexible y la mente como un producto social maleable está mal planteada. La concepción según la cual la estructura psicológica heredada «restringe» significaría que sin esta estructura seríamos más flexibles o maleables, o que tendríamos mayor capacidad de respuesta al entorno. Esto no sólo es falso, sino también absurdo. Sin esta estructura evolucionada no tendríamos capacidad de respuesta ambien-

27. El intento más sobresaliente de conectar biología y socialidad fue la sociobiología. Aunque cabría distinguir entre fases y líneas diversas de ese programa de investigación, todas tuvieron un trasfondo conceptual y metodológico común. Para dos de las mejores críticas al programa de investigación sociobiológico, véanse Rosenberg (1981) y Kitcher (1985). Para un análisis de cómo algunos sociobiólogos (por ejemplo, Lumsden y Wilson, 1981) tomaron acríticamente el supuesto de equipotencialidad de la mente humana, cfr. Janicki y Krebs (1998). 
tal (es decir, a partes seleccionadas del entorno de una forma organizada) contingente. Los mecanismos evolucionados no impiden, restringen o limitan al sistema para hacer cosas que en su ausencia podrían hacerse. Nuestras adaptaciones cognitivas evolucionadas — nuestros mecanismos psicológicos heredados - son los medios por los que canalizamos o creamos todo el complejo de actividades humanas. La estructura evolucionada no restringe en este sentido; crea o capacita.

En segundo lugar, el modelo fundado en un sistema de procesamiento de información equipotencial se basa en una psicología imposible. Los resultados empíricos de las últimas décadas de la psicología cognitiva, la biología evolucionaria, la inteligencia artificial, la psicología del desarrollo y la lingüística, entre otras, convergen en una misma conclusión: una arquitectura cognitiva que consistiera solamente en mecanismos equipotenciales, de uso general o independientes del contenido no podría realizar las tareas que sabemos que la mente humana realiza, o resolver los problemas adaptativos que los humanos resuelven (desde la orientación espacial hasta el aprendizaje de una lengua, pasando por el reconocimiento de las expresiones emocionales y las más variadas actividades de aprendiaje en distintos dominios). Los humanos, expuestos durante miles de años - particularmente, durante la última gran presión selectiva ocurrida en el Pleistoceno en sociedades de cazadores y recolectoresa patrones estables de información del entorno físico y social, habrían incorporado una colección de mecanismos dominio-específicos que operarían integradamente con algunos mecanismos dominio-generales (de prueba y error, inferenciales, etc.) por motivos de "economía» (en general, la evolución tiende a seleccionar aquellos rasgos y mecanismos que son más eficientes y a descartar los más «costosos») y "computacionales» (un procesador equipotencial que, por definición, no dispone ex ante de la capacidad de discriminar entre distintos patrones de información se paraliza ante la efectiva complejidad del mundo, de modo que la evolución habría seleccionado más favorablemente aquellos mecanismos especializados en procesar información específica, estable durante la evolución humana ${ }^{28}$. De modo que en el supradominio de la socialidad, el impacto sostenido de relaciones humanas intraculturales e interculturales recurrentes en entornos ancestrales favoreció la selección de mecanismos de resolución de problemas de la mente humana para explotar la estructura común de las entidades culturales y simbólicas. Entonces, las arquitecturas cognitivas humanas están "preequipadas» con mecanismos especializados que «saben» muchas cosas acerca de los humanos, sus relaciones sociales, sus emociones y expresiones faciales, la organización subyacente de las acciones sociales contingentes como las amenazas, los intercambios, el lenguaje, la motivación, etc.

28. Para una panorámica del inabarcable caudal de trabajos sobre la modularidad, y sobre las discusiones entre modularidad dominio-específica y modularidad dominio-general, cfr. Hirschfeld y Gelman (1994), Fodor (1983; 2000), Cosmides y Tooby (1994b) y Carruthers y Chamberlain (2000). 
Lo cual ayuda a entender, en tercer lugar, que la lógica de la explicación de un sistema de procesamiento equipotencial o de uso general se fundamentaría en conceptos incorrectos y meramente intuitivos tomados de teorías del desarrollo periclitadas. Por ejemplo, que rasgos de la organización mental adulta no estén presentes en la psicología infantil temprana no implica que no haya una arquitectura cognitiva evolucionada. Del mismo modo que la dentición está ausente en el nacimiento pero aparece en el proceso de maduración, también los mecanismos psicológicos y módulos cognitivos pueden desarrollarse durante el ciclo vital, particularmente en sus primeras fases. Repárese en que es condición necesaria para el desarrollo de la dentición el que el organismo infantil ingiera, por ejemplo, calcio. Por lo mismo, los módulos cognitivos ontogenéticos de carácter relacional, social y simbólico no podrían desarrollarse apropiadamente si en las primeras etapas del desarrollo cognitivo no recibieran estímulos del contexto social. De modo que, interesantemente, la perspectiva integrada de la psicología evolucionaria permite comprender que la cognición, en muchos dominios, es irreductiblemente social, requiere de interacción humana, entorno institucional y socialización en distintos grados. Lo cual converge en la idea de que no puede entenderse la cognición humana ni como un mero efecto de la socialización masiva, ni como una estructura que desarrolla mecanismos y dinámicas asociales. Por esta razón, el que la mayor parte de los rasgos de la organización mental adulta no estén presentes ya en el nacimiento no tiene que explicarse causalmente sólo por la exposición a la cultura transmitida, sino que también puede $-\mathrm{y}$ debe- haber otras vías causales no consideradas en los análisis tradicionales. Hoy disponemos de una base empírica suficiente para poder afirmar que los niños son capaces de categorizar muchos dominios de la realidad a muy temprana edad, lo cual haría insostenible un enfoque basado únicamente en la socialización y, por ende, en el aprendizaje. Recientes investigaciones transculturales convergen en apuntar que todos los humanos tenemos un conjunto de capacidades mentales básicas que facilitan un acceso diferenciado y solapado a distintos dominios de la naturaleza: la mecánica intuitiva o folk mechanics (sobre los límites o contornos de los objetos y sus movimientos), la biología intuitiva o folk biology (sobre las configuraciones de las especies biológicas y sus relaciones) y, también, la folk psychology $y^{29}$.

La folk psychology constituiría un caso paradigmático de cómo es posible reconducir estas tres críticas. La psicología evolucionaria permite explorar una vía alternativa a la conceptualización (dicho de otro modo: una reconceptua-

29. Existe una vasta literatura teórica y empírica sobre la existencia de módulos cognitivos ontogenéticos, capacidades cognitivas innatas de clasificación de la realidad (física, biológica, humana, social...), aprendizaje orientado por mecanismos cognitivos no aprendidos, etc. Desde los trabajos pioneros de Eleanor Rosch (1978) y otros, la relación de bibliografía relevante es inacabable. Para una panorámica de los trabajos que dan cuenta de las principales investigaciones, cfr. Atran (1990), Hirschfeld y Gelman (1994), Sperber y otros (1995), Pinker (1997), Geary y Huffman (2002) . 
lización) de los deseos y las creencias, que son variables inherentmente inobservables $^{30}$. Si los deseos o las creencias no son sólo el producto de la socialización y han emergido en todas las culturas, entonces la hipótesis causal más verosímil es que nuestros programas de desarrollo o arquitecturas cognitivas nos imponen esta forma de interpretar el mundo interno de los demás humanos. Las inferencias sobre los estados mentales de los demás serían generadas por un sistema cognitivo dominio-específico a veces denominado «módulo de la teoría de la mente» (Leslie, 1987) ${ }^{31}$. El módulo consistiría en un mecanismo computacional especializado que capacitaría para representarse la noción de que los «agentes» pueden tener «actitudes» hacia las "proposiciones» (entonces, Tristán puede «creer» que $X$, Isolda puede «pensar» que $X$, etc.). Este sistema inferencial dominio-específico se desarrolla siguiendo una pauta característica que es común en los distintos contextos culturales estudiados ${ }^{32}$. Las personas de distintas culturas pueden desplegar sus psicologías intuitivas de formas diversas (la expresión y percepción de estas intuiciones es, en parte, modelada socialmente), pero la maquinaria computacional que guía el desarrollo de las nociones intuitivas será la misma, y, de hecho, algunas de las nociones desarrolladas también serán las mismas. Así, los humanos accederíamos al mundo con la propensión a organizar nuestra comprensión de las acciones de los demás en términos de deseos y creencias, del mismo modo que el repertorio de nuestra retina organiza patrones en dos dimensiones bajo el supuesto de que el mundo es tridimensional y de que los objetos son permanentes, tienen contornos y son sólidos. Entonces, no sólo los mecanismos evolucionados suponen que ciertas cosas de la vida social tenderán a ser ciertas, sino que esos procedimientos especializados, formatos representacionales, estímulos y sistemas de categorización imponen - a partir de un conjunto infinito de alternativas potenciales - una elaborada y detallada organización sobre la experiencia compartida por todos los miembros estadísticamente normales de nuestra especie.

Este enfoque basado en el procesamiento de información realizado de forma específica por mecanismos cognitivos evolutivamente diseñados por su adaptación a patrones estables de la realidad tiene — más allá de las importantes discusiones sobre el alcance de la dominio-especificidad- serias consecuen-

30. Razón por la cual se ha puesto en entredicho su propia existencia (y no sólo por parte del conductismo). Para una exposición clásica de los argumentos a favor de considerar elementos proposicionales como deseos y creencia como parte de la realidad, véase Dennett (1991). Para una versión "eliminacionista» de los mismos, véase Churchland (1991).

31. En efecto, una intensa investigación en el campo del desarrollo cognitivo recientemente ha proporcionado un apoyo substancial a la hipótesis de que nuestra arquitectura cognitiva evolucionada incluye procesos que causan que los niños de muy corta edad desarrollen verosímilmente una folk psychology o "teoría de la mente». Cfr. Astington y otros (1988); Baldwin y Moses (1996); Bartsch y Wellman (1989); Carey (1985); Carey y Gelman (1991); Humphrey (1987); Keil (1989); Leslie (1987, 1995); Malle y otros (2001); Markman (1989); Perner (1991); Premack (1995); Repacholi y Gopnik (1997); Spelke (1988), y Wellman (1990).

32. Además, la literatura que ilustra la existencia de daños cognitivos selectivos como el autismo reforzaría la concepción modular en este dominio (cfr. Baron-Cohen, 1995). 
cias para la investigación en ciencia social, puesto que los mecanismos de procesamiento de información dominio-específicos generarian parte del contenido concreto de la socialidad y la cultura humanas, incluidas ciertas conductas, artefactos y representaciones lingüísticamente transmitidas; y el contenido social y cultural generado por éstos y otros mecanismos estaría disponible para ser adoptado o modificado por los mecanismos psicológicos de otros miembros de la población ${ }^{33}$. Puesto que estos mecanismos no operarían autárquicamente, sino que, precisamente por su conformación social a través de la evolución, presuponen un trasfondo interactuante, esta vía investigadora abre las puertas a unas nuevas formas de concebir la transmisión cultural, con hipótesis teóricas muy prometedoras ${ }^{34}$.

Esta reconceptualización de la relación entre la mente y el mundo social presenta caminos inéditos de exploración, categorización y explicación causal de la realidad social. Por lo pronto, aumentar el conocimiento sobre nuestra arquitectura cognitiva fija restricciones más exigentes sobre las teorías sociales admisibles, lo cual no implica que toda investigación social realizada hasta ahora bajo el supuesto tácito de una mente como procesador de información equipotencial e ilimitadamente moldeable por el ambiente, tenga que desecharse. En muchos casos, lo contrario es lo cierto. En ciencia social, disponemos de un registro empírico extraordinario sobre cómo son los humanos y sus sociedades, y a la vez disponemos de teorías de rango medio que apuntan interesantes explicaciones causales. Sin embargo, la reconexión disciplinaria que conlleva el programa de investigación de la psicología evolucionaria proporciona un nuevo marco interpretativo que debería permitir reconciliar muchos de los valiosos datos empíricos disponibles con un patrón psicológicamente más realista ${ }^{35}$. Los fenómenos humanos descritos y categorizados solamente

33. Para la comprensión de la existencia de fenómenos unitarios subyacentes a la diversidad cultural manifiesta, véase Brown (1991; 1999).

34. Cabe destacar especialmente el desarrollo de la concepción de la evolución cultural como un modelo de herencia dual de Boyd y Richerson (1985) y Durham (1991); los trabajos pioneros de la "teoría memética» de Dawkins $(1976 ; 1986)$ y los modelos cuantitativos de Cavalli-Sforza y Feldman (1981); el modelo de coevolución de Barkow (1989; 2006); la concepción metacultural de la psicología cognitiva de Tooby y Cosmides (1992), y, por acabar en algún sitio, la línea de investigación sobre epidemiología de las creencias de Sperber (1994; 1996) y el modelo de ontologías intuitivas en las representaciones culturales de Boyer (1994). Éstos últimos, a su vez, han dado pie a innumerables investigaciones en el campo específico de las representaciones religiosas — una muestra significativa de las mismas son los números especiales de las revistas Behavioral and Brain Sciences, 2004, 27 (6) y Evolution and Cognition, 2004, 10, (1). Muy en otra línea, cabría destacar la aportación de Searle (1997), según la cual los humanos creamos realidad «institucional» a partir de una cognición equipada para ello, de modo que existiría una suerte de continuum entre lo biológico y lo institucional. Aunque se trata de una línea de investigación pendiente de mayor desarrollo, exhibe aspectos muy promisorios en punto a integración conceptual.

35. El criterio de realismo tiene que ver con la robustez del registro empírico disponible, que está vinculada a la posibilidad de contraste de los resultados de una disciplina con las aportaciones relevantes de otras ciencias adyacentes. Para una reinterpretación muy poderosa de los resultados de la sociología y la antropología, en términos de hipótesis sobre la existencia 
mediante el patrón interpretativo de las conductas manifiestas han conducido a que la actividad social humana tradicionalmente haya aparecido a los ojos de los científicos sociales como infinitamente variable, un haz caleidoscópico de hechos erráticos y volátiles que hacen enormemente difícil el descubrimiento de regularidades subyacentes, lo cual ha llevado a innumerables callejones sin salida teóricos y a la creación de una jerga científica completamente refractaria a cualquier intento de convergencia conceptual dentro de la propia disciplina y a la integración conceptual con otros campos científicos adyacentes. La promesa de la psicología evolucionaria es la de aportar un esquema interpretativo consistente en una matriz inconsútil de causalidad. Romper esta matriz equivale a aceptar y perpetuar el dualismo, y a optar por un modelo causalmente fragmentario en el que es admisible - y puede presentarse incluso como inevitable - el aislamiento de las distintas áreas de investigación.

Pero, por ahora, sólo se trata de una promesa. De cumplirse, el problema de qué descripción psicológica deben tomar las ciencias sociales para elaborar sus teorías dejaría de ser una falsa elección dicotómica entre puras disposiciones intuitivas y teorías científicas. Disponemos de una herramienta analítica potencialmente capaz de abordar la compleja realidad de la cognición humana - intencional y no intencional - tratando de conectarla causalmente con el resto de fenómenos del mundo. Sin caer en la burda estrategia conductista, la psicología evolucionaria permite tratar de evitar los problemas de la circularidad explicativa de un enfoque intuitivo mediante el estudio del diseño funcional evolucionado de la mente humana. Porque de lo que se trata es de rescatar para las ciencias sociales la complejidad de la naturaleza humana y de explicar su increíble potencial de creación de diversidad sociocultural, para lo cual no caben las estrategias teóricas y metodológicas procrusteanas, sino las que, de manera realista, aceptan esa complejidad como punto de partida.

\section{Referencias bibliográficas}

ARistóteles. Política. Traducción al castellano: Julián María y María Araújo. Madrid: Centro de Estudios Constitucionales.

Astington, J.W.; Harris, P.L.; Olson, D.R. (eds.) (1988). Developing Theories of Mind. Cambridge: Cambridge University Press.

Atran, S. (1990). Cognitive Foundations of Natural History. Cambridge: Cambridge University Press.

- (2005). «Adaptationism for human cognition: Strong, spurious or weak?». Mind \& Language, 20, 1, p. 39-67.

BALDWIN, D.A.; MOSES, L.J. (1996). «The ontogeny of social information gathering». Child Development, 67, p. 1915-1939.

de cuatro modelos exhaustivos de socialidad humana que podrían corresponderse con cuatro patrones cognitivos estructuralmente distintos de procesamiento de información social (comunidad, autoridad, parigualdad y proporcionalidad), véase Fiske (1991). 
BARKOW, J. (ed.) (2006). Missing the Revolution. Darwinism for Social Scientists. Oxford: Oxford University Press.

BARKow, J.; Cosmides, L.; ToOBY, J. (eds.) (1992). The Adapted Mind: Evolutionary Psychology and the Generation of Culture. Oxford: Oxford University Press.

BARON-CoHen, S. (1995). Mindblindness: An Essay on Autism and Theory of Mind. Cambridge, MA: The MIT Press.

BARTSCH, K.; Wellman, H.M. (1989). «Young children's attribution of action to beliefs and desires». Child Development, 60, p. 946-964.

Berger, P.; LuCKMANN, T. (1967). The social construction of reality. Nueva York: Anchor Books.

Blaug, M. (1997). Economic Theory in Restrospect. 5a. ed. Cambridge: Cambridge University Press.

Boyd, R.; Richerson, P.J. (1985). Culture and the Evolutionary Process. Chicago: The University of Chicago Press.

BOYER, P. (1994). The Naturalness of the Religious Ideas: A Cognitive Theory of Religion. Berkeley: University of California Press.

Brown, D.E. (1991). Human Universals. Nueva York: McGraw-Hill.

- (1999). «Human Nature and History». History and Theory, 38, 4, p. 138-157.

Butos, W.N. (2003). «Knowledge Questions: Hayek, Keynes, and Beyond». The Review of Austrian Economics, 16, 4, p. 291-307.

CAREY, S. (1985). «Constraints on semantic development». En: MeHLER, J.; Fox, R. (eds.). Neonate cognition. Hillsdale: Lawrence Erlbaum, p. 391-398.

CAREY, S.; Gelman, R. (eds.) (1991). The Epigenesis of Mind. Hillsdale, NJ, Lawrence Erlbaum.

Carruthers, P.; Chamberlain, A. (eds.). Evolution and the human mind. Modularity, language and meta-cognition. Cambridge: Cambridge University Press.

CAVAlli-SFORZA, L.L.; FeldMAN, M.W. (1981). Cultural Transmision and Evolution: A Quantitative Approach. Princeton: Princeton University Press.

CHOMSKY, N. (1959). «Review of Skinner's Verbal Behavion. Language, 35, p. $26-58$.

- (1975). Proceso contra Skinner. Barcelona: Anagrama.

Churchland, P. (1991). «Eliminative Materialism and the Propositional Attitudes». En: Boyd, R.; Gasper, Ph.; Trout, J.D. (eds.). The Philosophy of Science. Cambridge, MA: A Bradford Book. The MIT Press, p. 615-630.

COSMIDES, L.; TOOBY, J. (1994a). «Beyond intuition and instinct blindness: toward an evolutionarily rigorous cognitive science». Cognition, 50, p. 41-77.

- (1994b). «Origins of domain specificity: The evolution of functional organization». En: HirSCHFELD, L.A.; Gelman, S.A. (1994). Mapping the Mind. Domain Specificity in Cognition and Culture. Cambridge: Cambridge University Press, p. 85-116.

Cummins, R. (1975). «Functional Analysis». Journal of Philosophy, 72.

DAWKINS. R. (1976). The Selfish Gene. Nueva York: Oxford University Press.

- (1986). The Blind Watchmaker. Londres: Penguin.

DennetT, D. (1981). «Skinner skinned». En: Brainstorms. Philosophical Essays on Mind and Psychology. Brighton: The Harvester Press, p. 53-70.

- (1987). The Intentional Stance. Cambridge, MA: The MIT Press.

- (1991). "Real Patterns». Journal of Philosophy, 88, 1, p. 27-51.

- (1995). Darwin's Dangerous Idea: Evolution and the Meaning of Life. Nueva York: Simon \& Shuster.

DomèneCH, A. (2002). «Algunos enigmas de la racionalidad económica». En: DíEZ CalzadA, J.A. (ed.). Los límites de la globalización. Barcelona: Ariel, p. 65-84. 
- (2005). «Relativismo, filosofía y ciencias sociales». En: EsTANY, A. (coord.). Filosofía de las ciencias. Enciclopedia Iberoamericana de Filosofía. Madrid: Trotta, p. 293-322.

Durham, W.H. (1991). Coevolution: Genes, Culture, and Human Diversity. Stanford, Ca.: Stanford University Press.

Durkheim, E. (1987/1895). Las reglas del método sociológico. Trad. Antonio Ferrer, Madrid: Akal.

Flanagan, O. (1991). The Science of the Mind. Cambridge, MA: The MIT Press. FODOR, J. (1983). The Modularity of Mind. Cambridge, MA: The MIT Press.

- (2000). The mind doesn't work that way: The scope and limits of computational psychology. Cambridge, MA: The MIT Press.

GEARY, D.; HufFMAN, K. (2002). «Brain and Cognitive Evolution: Forms of Modularity and Functions of Mind». Psychological Bulletin, 128, p. 667-698.

GeHlen, A. (1966). Der Mensch: Seine Natur und seine Stellung in der Welt. Frankfurt am Main: Athenäum.

GigerenZer, G. (1991). «From tools to theories: A heuristic discovery in cognitive psychology». Psychological Review, 98, p. 254-267.

Goldman, A. (1993). «The Psychology of Folk Psychology». The Behavioral and Brain Sciences, 16: 15-28.

Hausman, D.M. (1992). The Inexact and Separate Science of Economics. Cambridge: Cambridge University Press.

HAYEK, F.A. (1973). "The Place of Menger's Grundsätze in the History of Economic Thought». En: HicKS, J.R.; WeBER, W. (eds.). Carl Menger and the Austrian School of Economics. Oxford: Clarendon Press.

Hempel, C. (1965). Aspects of Scientific Explanation and Other Essays in the Philosophy of Science. Nueva York: The Free Press.

Hirschfeld, L.A.; Gelman, S.A. (1994). Mapping the Mind. Domain Specificity in Cognition and Culture. Cambridge: Cambridge University Press.

Horgan, T.; WoOdWARd, J. (1985). «Folk Psychology is Here to Stay». Philosophical Review, 94, p. 197-226.

Humphrey, N. (1987). The Inner Eye. Faber \& Faber.

JANICKI, M.G.; KREBS, D.L. (1998). «Evolutionary Approaches to Culture». En: CRAWFORD, Ch.; KREBS, D.L. (eds.). Handbook of Evolutionary Psychology. Mahwah, NJ: Lawrence Erlbaum.

Karmiloff-Smith, A. (1992). Beyond Modularity: A Developmental Perspective on Cognitive Science. Cambridge, MA: The MIT Press.

KeIL, F.C. (1989). Concepts, kinds, and cognitive development. Cambridge, MA: The MIT Press.

KITCHER, Ph. (1985). Vaulting Ambition. Sociobiology and the Quest for Human Nature. Cambridge, MA: The MIT Press.

LESLIE, A.M. (1987). «Pretense and representation: The origins of «theory of mind». Psychological Review, 94, p. 412-426.

- (1995). "A theory of agency». En Sperber, D. y otros (eds.). Causal Cognition. Oxford: Clarendon Press.

Malle, B.F.; Moses, L.J.; BAldwin, D.A. (eds.). Intentions and intentionality. Foundations of social cognition. Cambridge, MA: A Bradford Book/MIT Press.

Markman (1989). Categorization and naming in children: Problems of induction. Cambridge, MA: The MIT Press.

Menger, C. (1981). The Principles of Economics. Nueva York: New York University Press. 
MundÓ, J. (2001). «Filosofia i epistemologia de les ciències socials». En MundÓ, J. (coord.). Filosofia de la ciència. Barcelona: Fundació per a la Universitat Oberta de Catalunya, p. 39-55.

- (2002). «El separatisme en la cultura científica». L'Espill, 11, p. 96-103.

- (2003). "Fundamentos psicológicos de la socialidad y la cultura humanas, y su importancia para su caracterización en otras especies». Ludus Vitalis, 19, p. 173-178.

Mundó, J.; RaVENTÓs, D. (2000). «Fundamentos cognitivo-evolucionarios de las ciencias sociales». Revista Internacional de Sociología, 25, p. 47-74.

Nagel, E. (1961). The Structure of Science. Problems in the Logic of Scientific Explanation. Londres: Routledge \& Keegan Paul.

PERNER, J. (1991). Understanding the representational mind. Cambridge, MA: The MIT Press.

PetitT, Ph. (1993). The Common Mind. Oxford: Oxford University Press.

PINKER, S. (1997). How the Mind Works. Nueva York: Norton.

- (2002). The Blank Slate: The Modern Denial of Human Nature. Nueva York: Viking.

Premack, D.; Premack, A. J. (1995). «Intention as psychological cause». En: SPERBER, D. y otros (eds.). Causal Cognition. Oxford: Clarendon Press.

Putnam, H. (1964). «Robots: Machines or Artificially Created Life». Journal of Philosophy, 61, 21.

- (1979). Scientific Realism and the Plasticity of Mind. Cambridge: Cambridge University Press.

Rabin, M. (1994). «Psychology and Economics». Journal of Economic Literature, 36, p. 11-46.

RaChlin, H. (1970). Introduction to Modern Behaviorism. San Francisco: W.H. Freeman. REPACHOLI, B.M.; GOPNIK, A. (1997). «Early reasoning about desires: Evidence from 14- and 18-months-old». Developmental Psychology, 33, p. 12-21.

RosCH, E. (1978). Cognition and categorization. Hillsdale, NJ: Lawrence Erlbaum.

- (1997). "Psicología cognitiva». En VARELA, FJ.; HAYWARD, J.W. (eds.). Un puente entre dos miradas. Santiago de Chile: Dolmen Ediciones.

Rosenberg, A. (1981). Sociobiology and the Preemption of Social Science. Oxford: Basil Blackwell.

- (1988). Philosophy of Social Science. Boulder: Westview Press.

- (1992). Economics. Mathematical Politics or Science of Diminishing Returns? Chicago: University of Chicago Press.

RudNer, R.S. (1966). «Functionalism and Other Problems of Teleological Inquiry». En: Philosophy of Social Science. Englewood Cliffs, N.J.: Prentice Hall Inc.

SAHLINS, M.D. (1976a). The use and abuse of biology: An anthropological critique of sociobiology. Ann Arbor: University of Michigan Press.

- (1976b). Culture and practical reason. Ann Arbor: University of Michigan Press.

SeARLE, J. (1997). La construcción social de la realidad. Traducción: Antoni Domènech. Barcelona: Paidós.

SKINNER, B.F. (1953). Science and Human Behavior. Nueva York: Free Press.

- (1974). About Behaviorism. Londres: Jonathan Cape.

SOBER, E. (1985). «Methodological behaviorism, evolution, and game theory». En: FetZER, J.H. (ed.). Sociobiology and Epistemology. Dordrecht: D. Reidel Publishing Company.

SPELKE, E.S. (1988). «The origins of physical knowledge». En: WEISKRANTZ, L. (ed.). Thought without language. Oxford: Clarendon Press, p. 168-184. 
SPERBER, D. (1994). "The modularity of thought and the epidemiology of representations». En: Hirschfeld, L.A.; Gelman, S.A. Mapping the Mind. Domain Specificity in Cognition and Culture. Cambridge: Cambridge University Press, p. 39-67.

- (1996). Explaining Culture. Cambridge, MA: MIT Press.

Sperber, D.; Premack, D.; Premack, A. (eds.). Causal Cognition. Oxford: Oxford University Press.

SPUHLER, J.N. (1959). The evolution of man's capacity for culture. Detroit: Wayne State University Press.

STICH, S.; NiCHOLS, S. (1992). «Folk psychology: Simulation or tacit theory?». Mind and Language, 7, 35-71; reimpreso en DAVIES, M.; STONE, T. (eds.). (1995). Folk Psychology: The Theory of Mind Debate. Oxford: Blackwell Publishers.

TAYLOR, C. (1964). Explanation of Behavior. Prometheus Books.

Thaler, R. (1987). «The Psychology of Choice and the Assumptions of Economics», reimpreso en (1991). Quasi Rational Economics. Nueva York: Russell Sage Foundation.

Tooby, J.; Cosmides, L. (1992). «The Psychological Foundations of Culture». En: BARKOW, J.; Cosmides, L.; ToOBY, J. (eds.). The Adapted Mind: Evolutionary Psychology and the Generation of Culture. Oxford: Oxford University Press.

VON Mises, L. (1981). Epistemological Problems of Economics. Traducción: George Reisman. Nueva York: New York University Press.

Von Neumann, J.; Morgenstern, O. (1944). Theory of Games and Economic Behavior. Princeton: Princeton University Press.

Wellman, H.M. (1990). The Child's Theory of Mind. Cambridge, MA: The MIT Press.

Wright, L. (1976). Teleological Explanations. University of California Press. 